Int. J. Dev. Biol. 56: 239-244

doi: $10.1387 / \mathrm{ijdb} .113482 \mathrm{mt}$

\title{
xCOUP-TF-B regulates $x$ Cyp26 transcription and modulates retinoic acid signaling for anterior neural patterning in Xenopus
}

\author{
MISAKI TANIBE ${ }^{1}$, SHO-ICHI ISHIURA ${ }^{1}$, MAKOTO ASASHIMA ${ }^{1,2}$ and TATSUO MICHIUE*,1 \\ ${ }^{1}$ Department of Life Sciences (Biology), Graduate School of Arts and Sciences, The University of Tokyo and \\ ${ }^{2}$ Research Center for Stem Cell Engineering, National Institute of Advanced Industrial Science and Technology (AIST), Japan
}

\begin{abstract}
Early embryogenesis in Xenopus development depends on correct anterior-posterior (A-P) neural patterning during gastrulation. It is known that high levels of retinoic acid (RA), a major intracellular signaling molecule, determine posterior cell fate, also reflecting an involvement in A-P neural patterning. Here we show that the known RA effector, xCOUP-TF, plays important roles in head development of Xenopus embryo. xCOUP-TF-B injection into the dorsal region of embryos induced formation of an abnormal head with small eyes. Analysis of brain marker gene expression revealed that XCOUP-TF-B injection induced slight anteriorization in embryos and attenuated the effects of RA treatment.This anteriorization effect was enhanced when xCOUP-TF-B was co-injected with $\mathrm{xCyp26A}$ or $\mathrm{xCyp26C}$, which are known RA metabolizing factors. Furthermore, $x$ COUP-TF-B injection enhanced $\mathrm{xCyp26A/C}$ transcription. Together, these results suggest that $\mathrm{xCOUP-TF}$ and xCyp26 are both regulated by Wnt signaling, and cooperatively function in RA signaling to affect A-P neural patterning.
\end{abstract}

KEY WORDS: Xenopus, retinoic acid, Wnt signaling, A-P patterning

Introduction

Axis determination is one of the most important events in embryonic patterning. During gastrulation, neural tissues are induced in ectodermal cells and, at the same time, anterior-posterior (A-P) neural patterning is determined. Several intracellular signaling pathways including Wnt and FGF play crucial roles in the determination ofA-P neural patterning (Niehrs, 1999; Sasai and DeRobertis, 1997).

Retinoic acid (RA) signaling is also important for precise A-P patterning, with an RA concentration gradient formed in early neurula (Chen et al., 1994). Further, RA treatment blocks head formation in Xenopus embryo, reflecting posteriorization (Durston et al., 1989; Ruiz i Altaba and Jessell, 1991), and alters hindbrain patterning (Papalopulu et al., 1991). Two RA nuclear receptors, RAR and RXR, are expressed in Xenopus embryos; these receptors associate with the regulatory region of target genes to promote target gene transcription (Ellinger-Ziegelbauer and Dreyer 1991: Brumburg et al., 1992).

Two factors are critical for adjusting the level of RA concentration along the A-P axis during patterning. The first is the RalDH family of genes, which function in RA synthesis. In Xenopus, RalDH2 is ex- pressed in the dorsal blastopore lip of the embryonic gastrula where it functions in brain development (Chen et al., 2001). Second, the Cyp26 genes are important for RAmetabolism (White et al., 1996), with xCyp26A, B, and C implicated in Xenopus embryogenesis (Hollemann et al., 1998; de Roos et al., 1999; Kudoh et al., 2002).

In a previous DNA microarray screen for novel genes that affect A-P neural patterning and that are regulated by canonical Wnt signaling (Tanibe et al., 2008; Michiue et al., 2007), we identified chicken ovalbumin upstream promoter transcription factor (COUP-TF) as a candidate gene (reviewed in Pereira et al., 1995). COUP-TF was first identified as a DNA-binding protein that interacts directly with the ovalbumin promoter (Pastorcic et al., 1986). COUP-TF encodes a steroid/thyroid hormone receptor that contains a DNAbinding domain including two zinc finger domains and one ligandbinding domain (LBD) (Evans, 1988). In Drosophila, COUP-TF is a causative gene of the seven-up mutation (Mlodzik et al., 1990), while two homologues of COUP-TF, xCOUP-TF-A and xCOUPTF-B, have been identified in Xenopus. In this study, it is shown

Abbreviations used in this paper: A-P, anterior-posterior; DA, dorso-animal; MO, morpholino oligo; RA, retinoic acid.

\footnotetext{
*Address correspondence to: Tatsuo Michiue. 3-8-1 Komaba, Meguro-ku, Tokyo 153-8902 Japan. Tel: +81-3-5454-6665. Fax: +81-3-5454-6665.

e-mail: tmichiue@bio.c.u-tokyo.ac.jp
} 
that $x$ COUP-TF-B is similar with mammalian COUP-TFII, and that xCOUP-TFA is distinct with xCOUP-TFI (Qiu et al., 1994; van der Wees et al., 1996). These genes are differentially expressed (van der Wees et al., 1996). For example, both genes are expressed in anterior neuroectoderm, but only COUP-TF-A is expressed in somite (van der Wees et al., 1996). The function of COUP-TF has been extensively studied, with some studies revealing an inhibitory role in RA signaling (Tran et al., 1992; Kliewer et al., 1992), and Xenopus COUP-TF implicated as a potential regulator of RA signaling in neural development (Schuh and Kimelman, 1995).

This study of XCOUP-TF function revealed important cooperative roles with $x$ Cyp26 in A-P neural patterning. $x$ COUP-TF-B is expressed in restricted sets of rhombencephalon at the neurula stage and affects the expression of several brain markers. The xCOUP-TF-B expression was dependent on Wnt signaling and enhanced anteriorization in cooperation with xCyp26. Moreover, xCOUP-TF was found to promote xCyp26 transcription. These data suggests that a novel mechanism of A-P neural patterning could exist via the regulation of RA signaling.
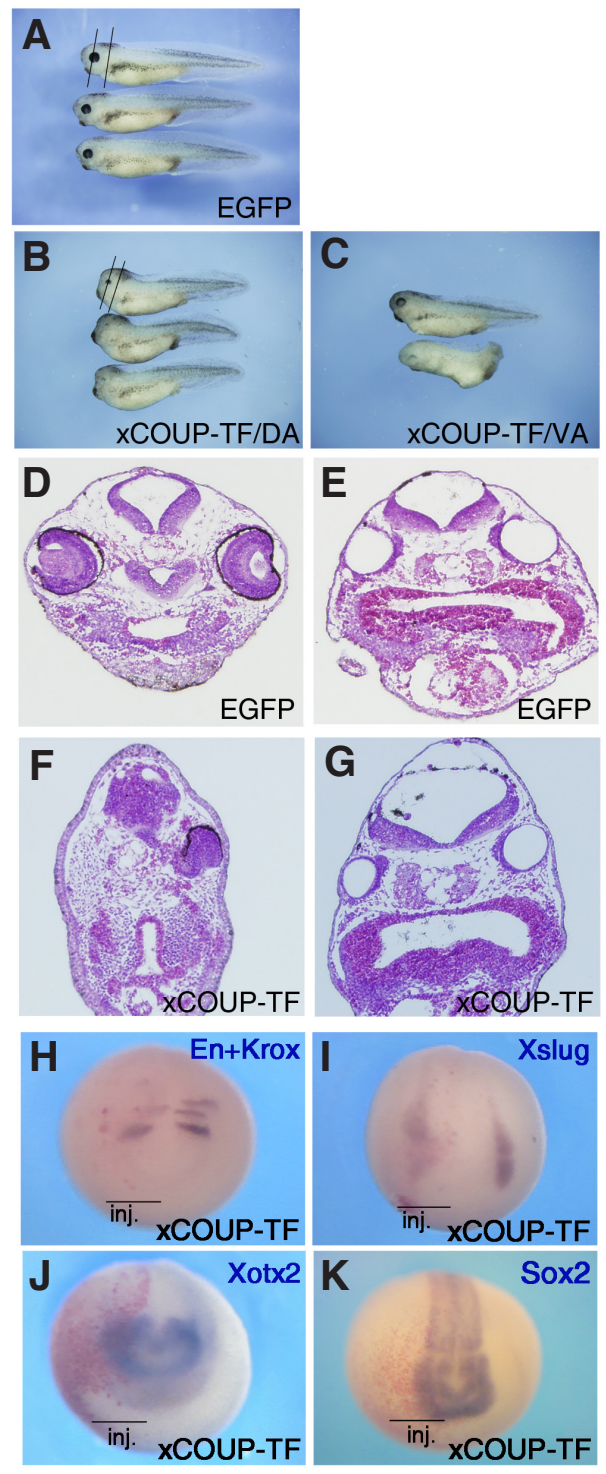

\section{Results}

\section{Microinjection with xCOUP-TF-B mRNA caused head defects} and misexpression of neural marker genes

As described in previous studies, dorsal injection with $x$ COUPTF-B mRNA disrupted anterior neural development (Schuh and Kimelman, 1995). To confirm this effect, we injected $x$ COUP-TF$B$ mRNA into the animal region of 4-8-cell embryos. Injection of $100 \mathrm{pg}$ of $x$ COUP-TF- $B$ mRNA into the dorso-animal (DA) region caused obvious head defects characterized by small eyes and a slightly bent axis (Fig. 1 A,B), while ventro-animal injections induced abnormalities in the abdominal region of tadpoles (Fig. 1C). We also observed transverse sections of the DA-injected embryos. Sections dissected at the eye vesicle showed that xCOUP-TF-B caused shrinkage of the diencephalon and loss of eye vesicles (Fig. $1 \mathrm{D}, \mathrm{F}$ ), and sections cut across the otic vesicles revealed hindbrain enlargement (Fig. 1 E,G).

In a previous study (Schuh and Kimelman, 1995), overexpression of $x$ COUP-TF-B mRNA altered the spatial expression of several genes such as En2 (a mid-hind brain marker), Krox20 (a rhomben-

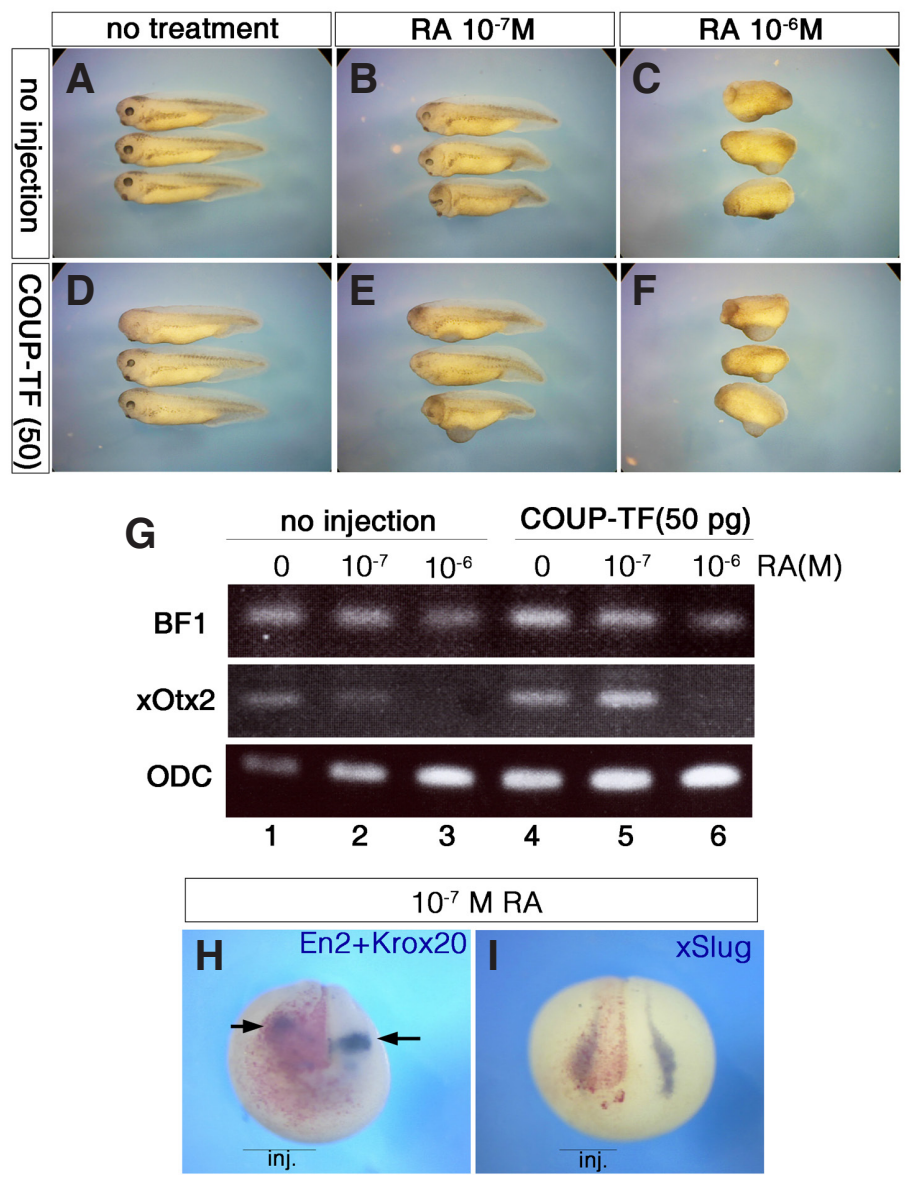

Fig. 2. COUP-TF-B could attenuate the retinoic acid (RA) effects. (A-F) Superficial phenotype of XCOUP-TF-B-injected embryos treated with $10^{-7}$ $M(\mathbf{B}, \mathbf{E})$ or $10^{-6} \mathrm{M}(\mathbf{C}, \mathbf{F})$ of $R A$. (G) RT-PCR analysis showing gene expressions of XBF1 (column 1) and Xotx2 (column 2). (H,I) Whole-mount in situ hybridization with XCOUP-TF-B-injected embryos treated with $10^{-7} \mathrm{M}$ $R A$. Injected side was marked with Red-gal staining by co-injection with lacZ mRNA. Transcription of En2 and Krox20 (H) or xSlug (I) were shown. 


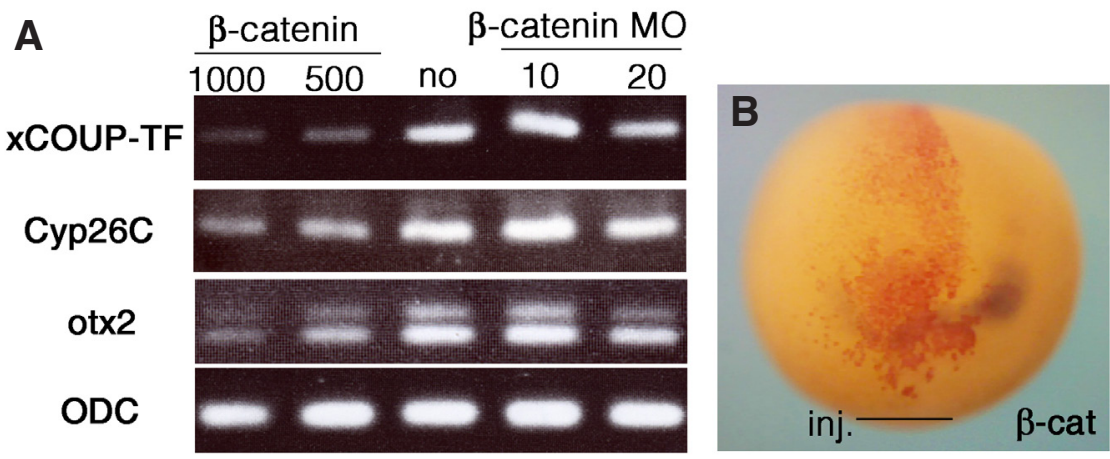

Fig. 3. Brain marker expression in $x$ COUP-TF-B-injected embryo. (A) RT-PCR analysis with cDNA derived from the anterior half of stage-13 embryo injected with 1000 pg (lane1) or $500 \mathrm{pg}$ (lane 2) of $\beta$ catenin $m R N A$, and 10 ng (lane 4) or 20 ng (lane 5) of $\beta$ cat MO. (B) Whole-mount in situ hybridization with $\beta$-catenin -injected embryo. For tracing the injected area, lacZ mRNA was co-injected and injected embryos were stained with Red-Gal.

cephalon marker), and Xtwist (a neural crest marker). To confirm these results, we performed whole-mount in situ hybridization. As described previously, $x$ COUP-TF-B MRNA injection disrupted En2 and Krox20 expression (Fig. 1H). Indeed, a complete absence of Krox20 expression (Fig. $1 \mathrm{H}$ ), while the pattern of En2 expression was slightly broadened and weakened (Fig. 1H). We also examined other neural markers. In xCOUP-TF-B injected embryo, xslug expression shifted posteriorly, suggesting anteriorization (Fig. 1I), although Xotx2 and Sox2 expressions were unchanged by $x$ COUP-TF- $B$ (Fig. $1 \mathrm{~J}, \mathrm{~K}$ ).

\section{XCOUP-TF-B attenuated posteriorization by RA}

Previous reports implicated an inhibitory role for XCOUP-TF in RAsignaling, and RA treatment is known to induce posteriorization in Xenopus embryo. We therefore examined whether xCOUP-TF could inhibit such an effect. First, we injected $x$ COUP-TF-B $m R N A$ into the DA region of 4-cell embryos, followed by treatment with RA solution at Stage 8. Without injection, treatment with $10^{-7} \mathrm{M}$ RA induced a slight head defect with small eye, while $10^{-6} \mathrm{M} R A$ treatment caused more severe defects (Fig. 2 B,C). In addition, the head shrinkage caused by treatment with RA was not rescued by the injection of $x$ COUP-TF-B mRNA (Fig. 2 E,F).

Next, we carried out RT-PCR analysis on these embryos. BF1 (telencephalon marker) and Xotx2 (forebrain marker) expressions were decreased with elevated RAconcentration (Fig. 2G, lane 1-3). Conversely, $x$ COUP-TF- $B$-injected embryos showed suppression of the $10^{-7} \mathrm{M}$ RA-induced decrease in BF1 and Xotx2 expression (Fig. 2G, lane 2 and 5). On the other hand, XCOUP-TF-B could not recover BF1 and Xotx2 expression in embryo with $10^{-6} \mathrm{M}$ RA treatment (Fig. 2G, lane 3 and 6).

We then assessed Krox20 and En2 expression further by in situ hybridization. Embryos treated with $10^{-7} \mathrm{M}$ RA showed a posterior shift in Krox20 expression in rhombomere 5 on the injected side (Fig. 2H), and this change was more apparent than in normal embryos without RA treatment (compare with Fig. 1H). Similar to En2/Krox20, the xslug expression area (neural crest marker) was also posteriorly shifted (Fig. $2 \mathrm{l}$ ). These results suggest that $x$ COUP-TF- $B$ attenuates posteriorization caused by RA.

\section{Canonical Wnt signaling downregulated expression of xCOUP-TF-B}

DNAmicroarray results suggested that $x$ COUP-TF-Bexpression is affected by canonical Wnt signaling. To confirm this, we carried out RT-PCR on $\beta$-catenin-injected embryos. $\beta$-catenin mRNAinjection decreased the level of $x$ COUP-TF- $B$ transcription, whereas $10 \mathrm{ng}$ of $\beta$-catenin $\mathrm{MO}$ injection slightly promoted $x$ COUP-TF- $B$ transcription, and $20 \mathrm{ng}$ injection weakly suppressed $x$ COUP-TF-B expression (Fig. 3A). Similar elevations were observed in $x$ Cyp26C and Xotx2 expression (Fig. 3A). The $\beta$-catenin-injected embryos also showed inhibition of spatial expression of $x$ COUP-TF-B (Fig. $3 \mathrm{~B})$. These data together suggests that $X C O U P-T F-B$ is negatively regulated by canonical Wnt signaling.

\section{Coinjection of xCOUP-TF-B with xCyp26C enhanced the anterior defect}

We also previously reported that $x$ Cyp26C is important for precise anterior neural patterning (Tanibe et al., 2008). Indeed, our microarray analysis identified both $x$ Cyp26C and $x$ COUP-TF-B as genes whose transcripts were more abundant in the anterior half of neurula than in the posterior half. Furthermore, it is known that both $x C y p 26 C$ and $x$ COUP-TF- $B$ negatively regulate RA signaling. We therefore hypothesize that $x C O U P-T F-B$ is involved with xCyp26C function.

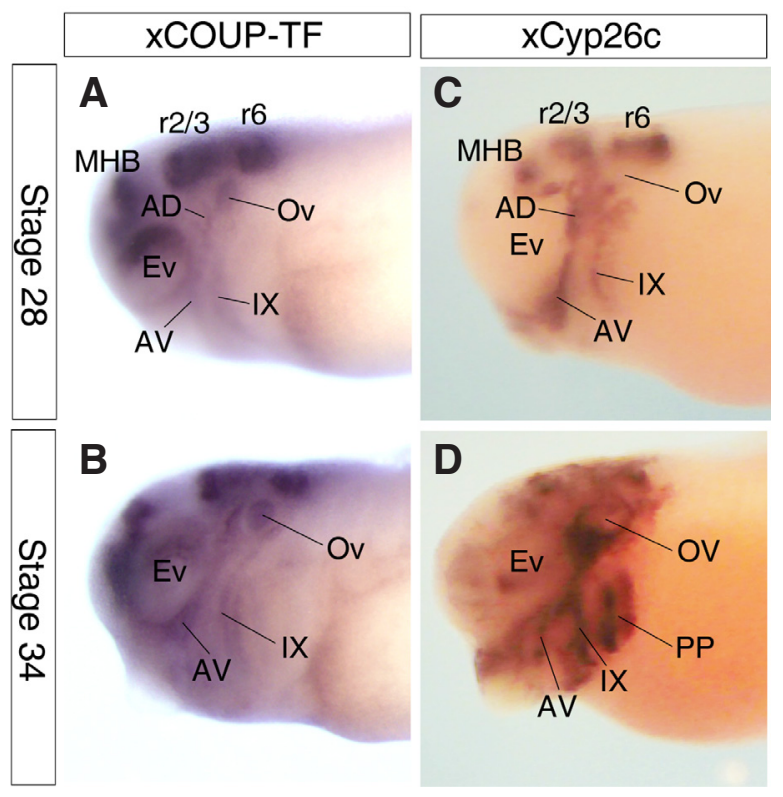

Fig. 4. Comparison of $x$ COUP-TF-B and $x C y p 26 C$ expression in brain region of tadpole. (A-D) Whole mount in situ hybridization was carried out with COUP-TF-B (A,B) and XCyp26C (C,D) probe. We observed Stage 28 $(\mathbf{A}, \mathbf{C})$ or Stage 34 (B,D) embryo. MHB: midbrain-hindbrain boundary. AD: anterodorsal lateral line pracode. AV: anteroventral lateral line pracode. Ev: eye vesicle. Ov: otic vesicle. IX: Epibranchial IX placode. PP: pharyngeal pouch. R2/3: rhombomere 2/3. R6: rhombomere 6. 
To assess this possibility, we first compared the spatial expression of $x$ COUP-TF-B with that of $x$ Cyp26C. As previously described, $x$ Cyp26C was expressed in restricted subsets of rhombomere and cranial placode (Fig. 4 C,D: Tanibe et al., 2008). xCOUP-TF showed a similar pattern of expression to that of $x$ Cyp26Cin rhombencephalon (Fig. $4 \mathrm{~A}, \mathrm{C}$ ), while both $x$ COUP-TF-B and $x$ Cyp26C expression was also seen in some sets of cranial placodal nerves, which were anterodorsal lateral line, anteroventral lateral line, and epibranchial IX (Fig. 4 A-D: Schlosser and Northcutt (2000); Baker and Bronner-Fraser (2001)). Although similar, there were some differences between the expression patterns of $x$ Cyp26C and $x$ COUP-TF-B. For example, these genes were differently expressed in each each placodal nerve (Fig. 4A-D), while only $x$ COUP-TF-B expression was observed in eye, otic vesicles, and telencephalic regions (Fig. $4 \mathrm{~A}, \mathrm{C}$ and Fig. $4 \mathrm{~B}$ and $\mathrm{D}$ ).

To further explore the expression of these genes, we carried out co-injection analyses using $x$ COUP-TF-B and $x$ Cyp26C. In

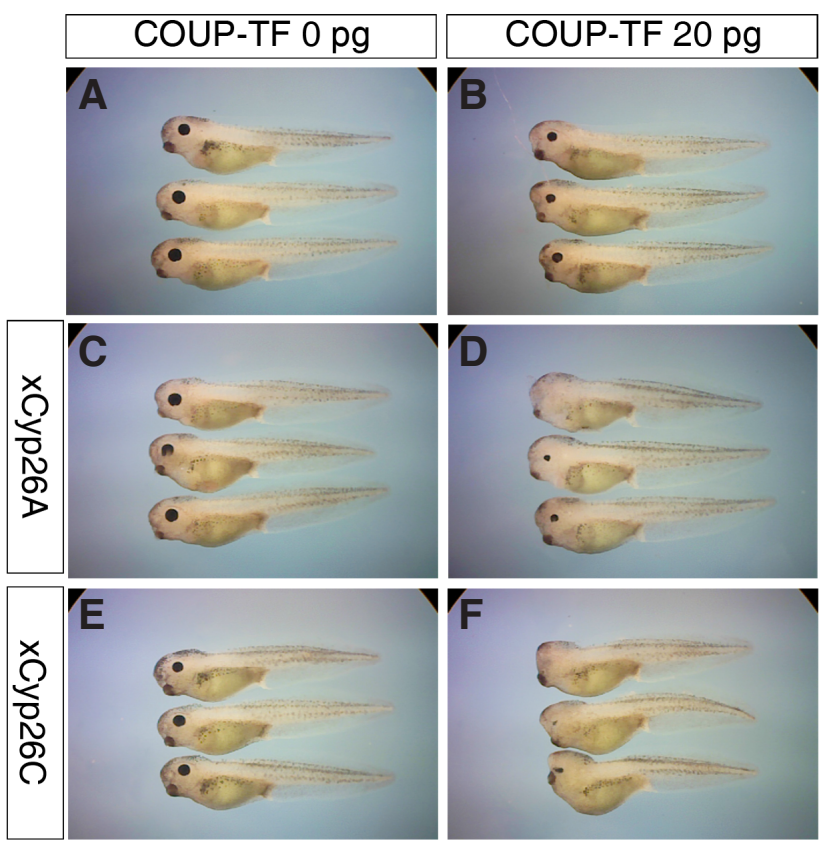

G

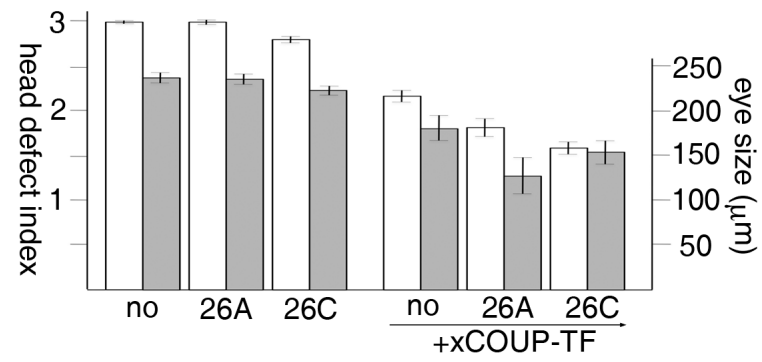

Fig. 5. Co-injection of $x$ COUP-TF-B enhanced the head defect phenotype induced by ectopic expression of $x$ Cyp26A or $x$ Cyp26C. (A-F) 500 pg of xCyp26A (C, D) or 500 pg of xCyp26C (E, F) was co-injected with XCOUP-TF-B (D, F), and injected embryos were observed at stage 40. (G) The open graph bar indicates head defect index (left column; Michiue et al., 2004) and gray bar indicates average eye size of injected embryo (right column). 26A and 26C indicates xCyp26A and xCyp26C, respectively. Error bar indicates S.E.
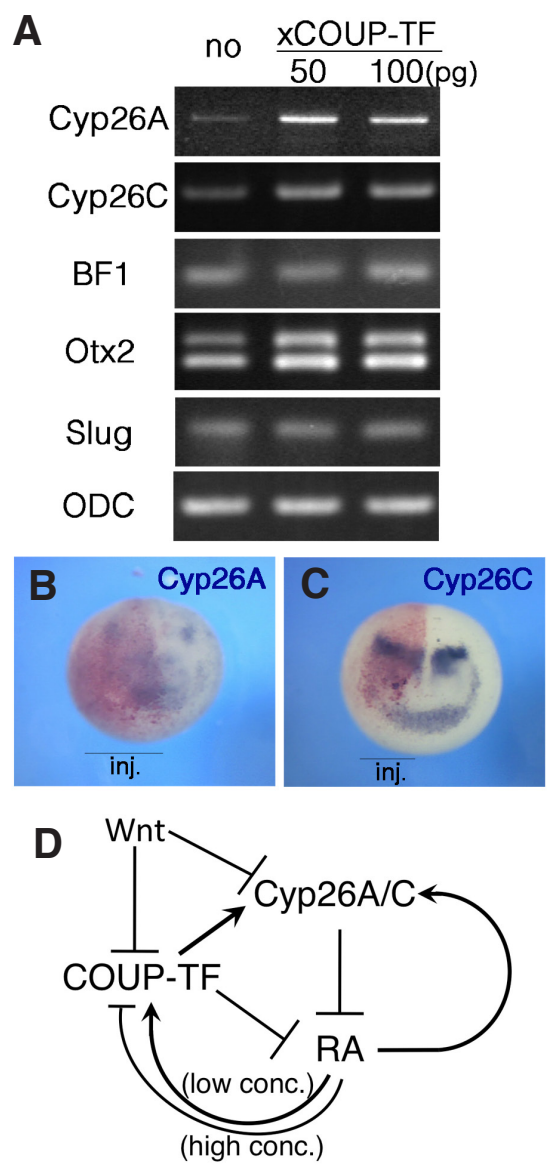

Fig. 6. $x$ COUP-TF-B enhanced transcription of both $x$ Cyp26A and xCyp26C. (A) RT-PCR was performed with stage-13 embryos injected with 50 pg (lane 2) or 100 pg (lane 3) of XCOUP-TF-B. We observed gene expression of Cyp26A (column 1), Cyp26C (column 2), XBF-1 telencephalon marker: column 3), Xotx2 (forebrain marker: column 4), xSlug (neural crest marker: column 5), and ODC (quantitative control: column 6). (B,C) Whole mount in situ hybridization of embryos injected with $75 \mathrm{pg}$ of XCOUP-TF-B with $\times$ Cyp26A $\left(B, B^{\prime}\right)$ or $x$ Cyp26C $\left(C, C^{\prime}\right)$. The injection side was marked with Red-Gal. (D) Schematic model of xCOUP-TF function.

this experiment, we also used xCyp26A, another xCyp26-family member. A low dose of $x C O U P-T F-B$ injected into the DA region of embryos caused minimal head defect (Fig. 5B). Similarly, injection of $250 \mathrm{pg}$ of $x$ Cyp26A showed normal phenotype (Fig. 5C). On the other hand, the co-injection of $x$ COUP-TF-B and $x$ Cyp26A induced a more marked head defect compared to that caused by $x$ COUP-TF-B alone (Fig. 5D). Co-injection with $x$ Cyp26C also showed similar effects on head defect (Fig. 5 E,F). More detailed examination confirmed that eye size was clearly reduced in the co-injected embryos (Fig. 5G).

xCOUP-TF-B increased $x$ Cyp26A and xCyp26C transcription

The co-injection analysis suggests that $x$ COUP-TF- $B$ could positively affect $x$ Cyp26A/C function. Previous results indicated a direct association between XCOUP-TF and RXR that negatively regulates $\mathrm{RA}$ signaling by competing with RAR (Kliewer et al., 1992). We therefore hypothesized that $x$ COUP-TF- $B$ regulates $x$ Cyp26A/C transcription. To test this, we examined whether $x$ COUP-TF- $B$ modulates the level of $x C y p 26 A / C$ expression. RT- 
PCR analysis revealed that $x$ COUP-TF-Boverexpression increased the expressions of both $x C y p 26 C$ and $x$ Cyp26A (Fig. 6A, 1st and 2nd column). Subsequent in situ hybridization of $x$ COUP-TF- $B-$ injected embryos indicated little change in the expressions of $x$ Cyp26A (Fig. 6B); however, xCyp26C expression increased in $x C O U P$-TF- $B$-injected areas (Fig. $6 \mathrm{C}$ ). These results suggest that $x$ COUP-TF- $B$ upregulates $x C y p 26 C$ transcription.

\section{Discussion}

This study showed that XCOUP-TF acts cooperatively with $x$ Cyp26A/C in A-P neural patterning of early embryogenesis. $X C O U P$-TF-Binjection increased the expression of several anterior neural genes, and suppressed RA-induced effects. Indeed, these results seemed to implicate an anteriorizing function for XCOUPTF. Ectopic expression of $x$ COUP-TF- $B$ caused eye defect and enlarged hindbrain formation. This result could reflect an anteriorization effects, but the inhibitory function of xCOUP-TF for RA signaling is dominant in hindbrain and the eye defect may reflect a separate function independent of RA signaling. In the previous report, $10^{-6} \mathrm{M}$ RA treatment suppressed COUP-TF-B expression in brain region (van der Wees et al., 1996). However, this result may reflect loss of COUP-TF expression area by treatment with higher RA concentration.

In this study, xCOUP-TF-B synergistically enhanced the superficial phenotype caused by $x$ Cyp26A/C, although because xCyp26A/C is not a transcription factor, xCOUP-TF-B and xCyp26A/C are unlikely to function in parallel as cofactor(s). However, our findings suggest that xCOUP-TF-B and xCyp26A/C may work in the same regulation network as described in Fig. 6D. Indeed, we showed that XCOUP-TF-B enhanced xCyp26A/C transcription, suggesting that $x$ COUP-TF-B physically interacts with the upstream region of $x$ Cyp26A/C gene. RA response element (RARE) sequences can be found upstream of the xCyp26A transcribed region, but not in that of xCyp26C (Tanibe et al., unpublished). To clarify our working hypothesis, further investigation is needed to identify the exact regulatory regions.

We also demonstrated the dependency on Wnt signaling in $x C O U P-T F-B$ transcription. Supporting the microarray analysis, $x$ COUP-TF- $B$ transcription was decreased by $\beta$-catenin-mRNA injection. Interestingly, the gene expressions of both $x C y p 26 \mathrm{~A} / \mathrm{C}$ and $x$ COUP-TF- $B$ are increased by the modulation of canonical Wnt signaling, suggesting crosstalk between Wnt and RA signaling. In particular, the fact that RA signaling factors are regulated by Wnt signaling may reflect epistasis of Wnt for RA signaling.

Based on the results of this study, we have proposed a model as follows (Fig. 6D). (1) Wnt signaling negatively regulates expression of both Cyp26A/C and $x$ COUP-TF-B. (2) RA signaling positively regulates both $x$ Cyp26A/C and xCOUP-TF-B. (3) xCOUP-TF-B positively regulates $x$ Cyp26A/C expression. (4) Both xCOUP-TF$B$ and xCyp26C negatively regulate RA signaling. In context of head specification, canonical Wnt signaling is negative regulated, followed by promotion of both xCOUP-TF-B and xCyp26A/C. We also propose that transcriptional enhancement of $x$ Cyp26A/C by $x$ COUP-TF-B will help to maintain low RA levels, and that ectopic expression of both $x$ COUP-TF-Band $x$ Cyp26A/C by exposure to RA could also be important for recovering normal RA level and precise anterior specification. Further extensive biochemical analyses are needed to clarify the molecular mechanisms underlying our model.

\section{Materials and Methods}

\section{DNA constructs}

DNA fragments of the $x$ COUP-TF-B gene were amplified by RT-PCR, and then ligated into pBluescriptSK(+) to make the pXCOUP-TF-B/SK construct. For the injection construct ( $\mathrm{pXCOUP-TF-B/CS)}$, we inserted an EcoRI-Xhol fragment of pXCOUP-TF/SK into pCS2(+).

\section{Microinjection}

Messenger RNAs for microinjection were synthesized using the mMessage mMachine SP6 kit (Ambion) and were cleaned up with an RNeasy RNA purification kit (QIAGEN). Transcribed mRNA was injected using a Picoinjector (Harvard Medical Instrument). Xenopusembryos were obtained by artificial fertilization and dejellied with $4.6 \%$ L-cysteine hydroxychloride. Injection was performed in 5\% Ficoll-Steinberg's solution, and injected embryos were cultured in the same solution for 5 hours, and then in 10\% Steinberg's solution until the appropriate developmental stage.

\section{In situ hybridization}

In situ hybridization was carried out as previously described (Tanibe et al., 2008). In brief, embryos were bleached in hydrogen peroxide-methanol before fixation in MEMFA solution (formaldehyde-MOPS solution) and dehydration with ethanol. Rehydrated embryos were hybridized with DIGlabeled probe for $24 \mathrm{~h}$ at $60^{\circ} \mathrm{C}$. Embryos were then incubated with $2000 \mathrm{x}$ anti-DIG antibody (Roche) for $12 \mathrm{~h}$, washed 5 times, and then visualized by reaction in NBT/BCIP solution.

\section{RT-PCR}

We used the Superscriptll reverse transcriptase (Invitrogen) for cDNA synthesis. Primers for PCR were as follows:

xCOUP-TF-B

$$
\text { 5'-CGAATCTTCCAAGAGCAG GTC -3' and }
$$
5'-TTA TTG TAT GGA CAT ATA GGG CC-3';

xCyp26c 5'-ACGAGG GGAAACTGGGCAAATTCAAC-3' and 5'-TCAGGCAAGTGACCCATTTCTTGC TGC-3';

xCyp26a 5'-CTTGCGGAGGTGGAGTGA GGT G-3' and 5'-GCTTAAATA GAGCTGGAGAAGGG-3';

Xotx2 5'-GGATGGATTTGTTACATCCGTC-3' and 5'-CACTCTCCGAGCTCACTTCCC-3';

XAG-1 5'-CATATGTGGTCCCAG TTATTTCCC-3' and 5'-CTCTTAGCGGTTTGAAACCGA-3';

BF1 5'-CATCGTTCAGTATCAACAGCC-3' and 5'-GACTCTCTGTCTTCACTTCCAGG-3';

En2 5'-ATGAGCAGAATAACAGGGAAGTGGA-3' and 5'-CCTCGGGGACATTGACTCGGTGGTG-3';

xSlug 5'-GGATACCCTTCATCTTTAGGTCG-3' and 5'-CATAAGCTGCACTGGAACTTCTC-3';

ODC 5'-GCCATTGTGAAGACT CTCTCCATTC-3' and 5'-TTCGGGTGATTCCTTGCCAC-3'.

For DNA amplification, we used PTC-200 thermal cycler (MJ Research / BioRad). In any cases, we performed 28 cycles of $\left(95^{\circ} \mathrm{C}, 15 \mathrm{sec} .-56^{\circ} \mathrm{C}\right.$, $15 \mathrm{sec} .-72^{\circ} \mathrm{C}, 30 \mathrm{sec}$.) chain reaction. Amplified DNA was detected by $\mathrm{EtBr}$ staining after agarose gel electrophoresis.

\section{Acknowledgment}

This work was supported in part by a Grant-in-Aid for Scientific Research from the Ministry of Education, Culture, Sports, Science and Technology of Japan (T. M) and from the Japan Society for the Promotion of Science for Young Scientists (M.T.).

\section{References}

BAKER CV, BRONNER-FRASER M. (2001). Vertebrate cranial placodes I. Embryonic induction. Dev Biol. 232: 1-61.

BLUMBERG B, MANGELSDORF DJ, DYCK JA, BITTNER DA, EVANS RM, DE 


\section{M. Tanibe et al.}

ROBERTIS EM. (1992). Multiple retinoid-responsive receptors in a single cell: families of retinoid "X" receptors and retinoic acid receptors in the Xenopus egg. Proc Natl Acad Sci USA 89: 2321-2325.

CHEN Y, POLLET N, NIEHRS C, PIELER T. (2001). Increased XRALDH2 activity has a posteriorizing effect on the central nervous system of Xenopus embryos. Mech Dev. 101: 91-103.

CHEN Y, HUANG L, SOLURSH M. (1994). A concentration gradient of retinoids in the early Xenopus laevis embryo. Dev Biol. 1994 161: 70-76.

DURSTONAJ, TIMMERMANS JP, HAGE WJ, HENDRIKS HF, DE VRIES NJ, HEIDEVELD M, NIEUWKOOP PD. (1989). Retinoic acid causes an anteroposterior transformation in the developing central nervous system. Nature 340: 140-144.

ELLINGER-ZIEGELBAUERH, DREYERC. (1991). A retinoic acid receptor expressed in the early development of Xenopus laevis. Genes Dev. 5: 94-104.

EVANS RM. (1988). The steroid and thyroid hormone receptor superfamily. Science 240: $889-895$

HOLLEMANN T, CHEN Y, GRUNZ H, PIELER T. (1998). Regionalized metabolic activity establishes boundaries of retinoic acid signalling. EMBOJ.17:7361-7372.

KLIEWER SA, UMESONO K, HEYMAN RA, MANGELSDORF DJ, DYCK JA, EVANS RM. (1992). Retinoid X receptor-COUP-TF interactions modulate retinoic acid signaling. Proc Natl Acad Sci USA. 89: 1448-1452.

KUDOH T, WILSON SW, DAWID IB. (2002). Distinct roles for Fgf, Wnt and retinoic acid in posteriorizing the neural ectoderm. Development 129: 4335-4346.

MICHIUE T, DANNO, H., TANIBE, M., IKUZAWA, M., ASASHIMA, M. (2007). Xenopus galectin-Vla shows highly specific expression in cement glands and is regulated by canonical Wnt signaling. Gene Expr Patterns 7: 852-857.

MLODZIK M, HIROMI Y, WEBER U, GOODMAN CS, RUBIN GM. (1990). The Drosophila seven-up gene, a member of the steroid receptor gene superfamily, controls photoreceptor cell fates. Cell 60: 211-224.

NIEHRS C. (1999). Head in the WNT: the molecular nature of Spemann's head organizer. Trends Genet. 15: 314-319.

PAPALOPULUN, CLARKEJD, BRADLEYL, WILKINSOND, KRUMLAUF R, HOLDER N. (1991). Retinoic acid causes abnormal development and segmental patterning of the anterior hindbrain in Xenopus embryos. Development 113: 1145-1158.

PASTORCIC M, WANG H, ELBRECHTA, TSAISY, TSAI MJ, O'MALLEY BW. (1986).
Control of transcription initiation in vitro requires binding of a transcription factor to the distal promoter of the ovalbumin gene. Mol Cell Biol. 6: 2784-2791.

PEREIRA FA, QIU Y, TSAI MJ, TSAI SY. (1995). Chicken ovalbumin upstream promoter transcription factor (COUP-TF): expression during mouse embryogenesis. $J$ Steroid Biochem Mol Biol. 53: 503-508.

QIU Y, COONEY AJ, KURATANI S, DEMAYO FJ, TSAI SY, TSAI MJ. (1994). Spatiotemporal expression patterns of chicken ovalbumin upstream promoter-transcription factors in the developing mouse central nervous system: evidence for a role in segmental patterning of the diencephalon. Proc NatlAcad SciU SA91:4451-4455

DE ROOS K, SONNEVELD E, COMPAAN B, TEN BERGE D, DURSTON AJ, VAN DER SAAG PT. (1999). Expression of retinoic acid 4-hydroxylase (CYP26) during mouse and Xenopus laevis embryogenesis. Mech Dev. 82: 205-211.

RUIZ I ALTABAA, JESSELL T. (1991). Retinoic acid modifies mesodermal patterning in early Xenopus embryos. Genes Dev. 5: 175-187.

SASAI Y, DE ROBERTIS EM. (1997). Ectodermal patterning in vertebrate embryos Dev Biol. 182: 5-20.

SCHLOSSER G, NORTHCUTT RG. (2000). Development of neurogenic placodes in Xenopus laevis. J Comp Neurol. 418: 121-146.

SCHUH TJ, KIMELMAN D. (1995). COUP-TFI is a potential regulator of retinoic acidmodulated development in Xenopus embryos. Mech Dev. 51: 39-49.

TANIBE M, MICHIUE T, YUKITAA, DANNO H, IKUZAWA M, ISHIURAS, ASASHIMA M. (2008). Retinoic acid metabolizing factor xCyp26c is specifically expressed in neuroectoderm and regulates anterior neural patterning in Xenopus laevis. Int J Dev Biol. 52: 893-901.

RAN P, ZHANG XK, SALBERT G, HERMANN T, LEHMANN JM, PFAHL M. (1992). COUP orphan receptors are negative regulators of retinoic acid response pathways. Mol Cell Biol. 12: 4666-4676.

VAN DER WEES J, MATHARU PJ, DE ROOS K, DESTRÉE OH, GODSAVE SF, DURSTON AJ, SWEENEY GE. (1996). Developmental expression and differential regulation by retinoic acid of Xenopus COUP-TF-A and COUP-TF-B. Mech Dev. 54: 173-184.

WHITE JA, BECKETT-JONES B, GUO YD, DILWORTH FJ, BONASORO J, JONES G, PETKOVICH M. (1997). cDNA cloning of human retinoic acid-metabolizing enzyme (hP450RAl) identifies a novel family of cytochromes P450. J Biol Chem. 272: 18538-18541. 


\section{Further Related Reading, published previously in the Int. J. Dev. Biol.}

Retinoid signalling is required for information transfer from mesoderm to neuroectoderm during gastrulation Ferran Lloret-Vilaspasa, Hans J. Jansen, Koen de Roos, Rosh A.S. Chandraratna, Maija H. Zile, Claudio D. Stern and Antony J. Durston Int. J. Dev. Biol. (2010) 54: 599-608

Molecular mechanisms controlling brain development: an overview of neuroepithelial secondary organizers

Claudia Vieira, Ana Pombero, Raquel García-Lopez, Lourdes Gimeno, Diego Echevarria and Salvador Martínez

Int. J. Dev. Biol. (2010) 54: 7-20

Retinoic acid metabolizing factor $\mathrm{xCyp26c}$ is specifically expressed in neuroectoderm and regulates anterior neural patterning in Xenopus laevis

Misaki Tanibe, Tatsuo Michiue, Akira Yukita, Hiroki Danno, Masayuki Ikuzawa, Shoichi Ishiura and Makoto Asashima

Int. J. Dev. Biol. (2008) 52: 893-901

The retinoic acid metabolising gene, CYP26B1, patterns the cartilaginous cranial neural crest in zebrafish

Susan Reijntjes, Adam Rodaway and Malcolm Maden

Int. J. Dev. Biol. (2007) 51: 351-360

Retinoic acid is required for specification of the ventral eye field and for Rathke's pouch in the avian embryo

Malcolm Maden, Aida Blentic, Susan Reijntjes, Sophie Seguin, Emily Gale and Anthony Graham Int. J. Dev. Biol. (2007) 51: 191-200

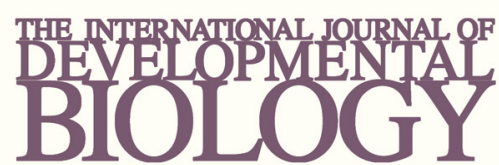

Volume 54 Nos. 6/7

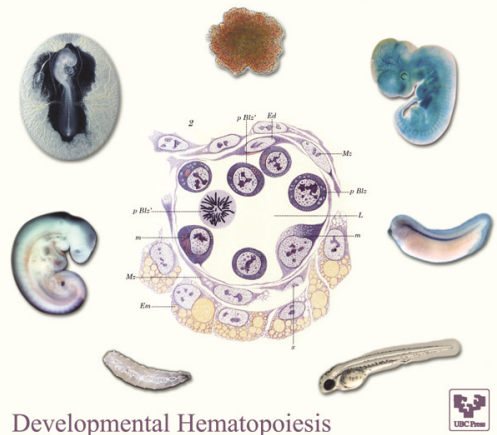

5 yr ISI Impact Factor $(2010)=2.961$

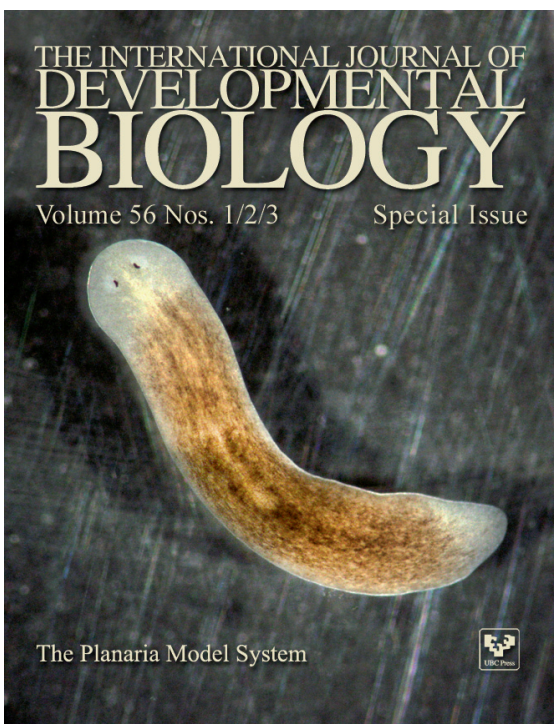

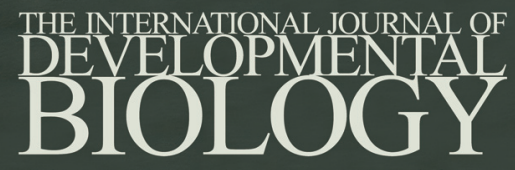
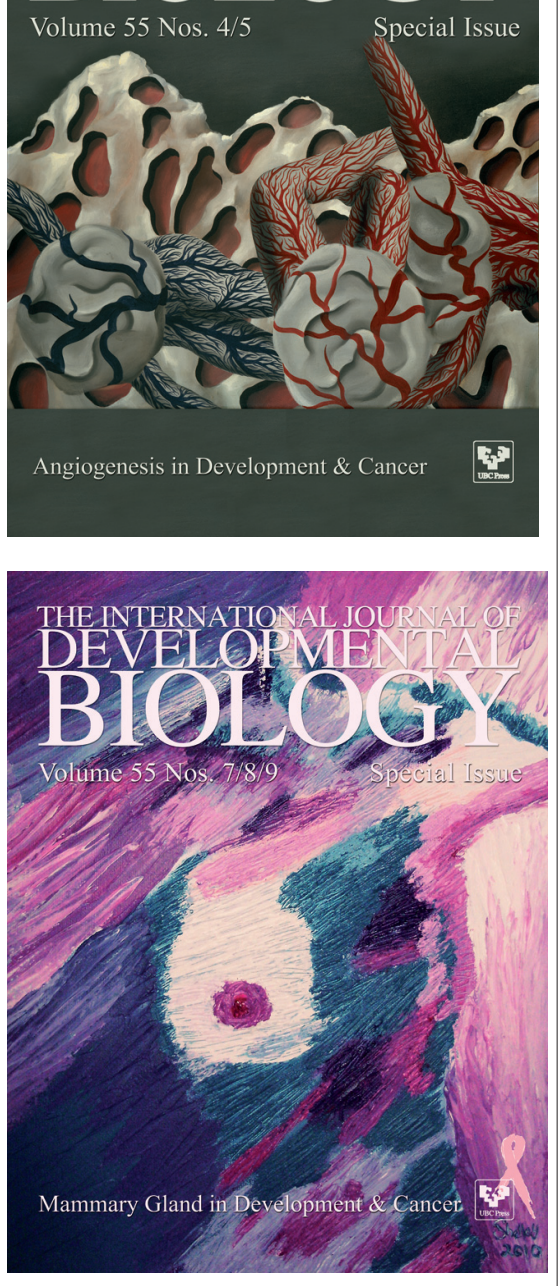